\title{
Análisis de la distribución espacial de los espacios públicos en las ciudades del Distrito Central, Comayagua y Siguatepeque, Honduras, 2017
}

Celina Michelle Sosa Caballero

\section{Resumen}

La identificación del patrón de distribución de los espacios públicos en las ciudades se analiza en el proyecto de investigación "Análisis Comparativo de la Distribución Espacial de los Espacios Públicos en las Ciudades del Distrito Central, Comayagua y Siguatepeque, Honduras". Para cumplir con el objetivo de la investigación, metodológicamente se han utilizado las herramientas de los sistemas de información geográfica para realizar un análisis y conocer las similitudes y diferencias que existen entre la distribución espacial de los espacios públicos de las ciudades en estudio. El resultado produjo que la distribución espacial de los espacios públicos en las tres ciudades presenta similitud en la categoría de infraestructura deportiva y recreativa, y diferencia en la categoría cultural.

Palabras Clave: Espacios Públicos, Patrones de distribución Geoespacial, Sistemas de Información Geográfica.

\section{Abstract}

The identification of the pattern of distribution of public spaces in cities is analyzed in the research project "Comparative Analysis of the Spatial Distribution of Public Spaces in the Cities of the Central District, Comayagua and Siguatepeque, Honduras". To realize the objective of the research, methodologically the tools of the geographic information systems have been used to carry out an analysis to know the similarities and differences that exist between the spatial distribution of the public spaces of the cities under study. The result produced of the public spaces in the three cities presents similarity in the category of sports and recreational infrastructure, and difference in the cultural category.

Keywords: Public spaces, Geospatial distribution patterns, Geographic Information Systems.

Celina Michelle Sosa Caballero

Honduras, Tequcigalpa M.D.C., Ciudad Universitaria, email: sosacelina@yahoo.com, Máster en Ordenamiento y Gestión del Territorio, ,Departamento de Ciencia y Tecnologías de la Información Geográfica, Facultad de Ciencias Espaciales. Universidad Nacional Autónoma de Honduras. 


\section{INTRODUCCIÓN}

La Agenda 21 Cultural en el tema de cultura, sostenibilidad y territorio define los espacios públicos como: "espacios de cultura". Así mismo, entre los principios de la Agenda de Ordenamiento Territorial del Consejo Centroamericano de Vivienda y Asentamientos Humanos -CCVAH- (2010), se contempla la calidad del espacio público y se definen de la siguiente manera: "Ios espacios públicos son elementos esenciales para la vida, dinamismo, identidad y cultura de un asentamiento humano, razón por lo que éstos deben ser diseñados y ubicados de forma que respondan a estas funciones. Deben articularse funcionalmente con la vivienda entre otros espacios, integrándose de tal forma que no se conviertan en lugares propicios para afectar la seguridad ciudadana" (CCVAH (2018)).

Los espacios públicos son tradicionalmente definidos como satisfactores de necesidades de recreación, ocio y culturales, y parte esencial en el desarrollo físico y emocional de las personas. Los municipios de Honduras forman parte de la iniciativa de la Agenda 21 Cultural, en la cual se declara el compromiso de "promover la existencia de los espacios públicos de la ciudad; fomentar su uso como lugares culturales de relación y convivencia y promover la preocupación por la estética de los espacios públicos y en los equipamientos colectivos" (Ciudades y Gobiernos Locales Unidos (2004)).

Entre los años del 2013 al 2016 se realizaron investigaciones sobre la distribución espacial de espacios públicos en las ciudades del Distrito Central, Comayagua y Siguatepeque, con los cuales, por primera vez en Honduras, se logran elaborar mapas de su distribución geoespacial, además del análisis de la concentración y dispersión de la infraestructura de dichos espacios en relación con el centro y las zonas residenciales para cada una de las ciudades estudiadas. 1

Una vez concluidos los estudios por ciudad, se ha valorado la oportunidad de avanzar analíticamente al proponer un estudio comparativo del comportamiento observado en cada una de las ciudades, que permita discutir sobre la posibilidad de encontrar un sólo patrón de distribución espacial de las infraestructuras de espacios públicos o si cada ciudad tiene una lógica particular de uso de suelo en cuanto a estos espacios. Obteniendo como resultado la identificación de similitudes en la categoría de infraestructura deportiva y recreativa, y diferencia en la categoría cultural.

\section{ANTECEDENTES}

Varios estudios sobre espacios públicos siguen la recomendación de la Organización Mundial de la Salud, en el sentido de que ciudades deben de tener como mínimo, de entre $10 \mathrm{~m}^{2}$ a $15 \mathrm{~m}^{2}$ de área verde por habitante, dispersados equitativamente en relación a la población (CAT-MED. (2018)).

En Europa, en la etapa de desindustrialización, la integración europea, la migración internacional, la globalización económica y el cambio climático, a los espacios públicos se les ve como apoyo a estos procesos (Madanipour y Degros

\footnotetext{
${ }^{1}$ Sosa Caballero, C. M. (2014). Distribución Geoespacial de los Espacios Públicos en el Distrito Central de Honduras. Sosa Caballero, C. M. (2016). Distribución Geoespacial de los Espacios Públicos en la Ciudad de Comayagua, Honduras. Sosa Caballero, C. M. (2016). Distribución de los Espacios Públicos en la Ciudad de Siguatepeque para el Análisis del Equilibrio Territoial de la Oferta Cultural y Recreativa de la Ciudad. Tegucigalpa, Honduras sin publicar.
} 
(2014)).

En Latinoamérica, actualmente se le da una gran importancia a la recuperación o construcción de espacios públicos, por tratarse de un lugar de esparcimiento y convivencia.

Esta es una de las razones por las cuales hay estudios que enfatizan sobre la recuperación de los citados espacios: Colombia, Venezuela, Chile y México son algunos de los países que han tratado el tema. Algunos de los estudios destacan lo siguiente:

- El estudio de Espació Público y Calidad Urbana en Pereira Visión de una Nueva Ciudad (Colombia), menciona que habitar una ciudad no solo consiste en obtener una propiedad de espacio privado, sino que, en una estructura adecuada de espacio público se observa el complemento necesario de sentido individual de habitar y es por esta razón que se debe de pensar y realizar intervención destinada a la mejora de la calidad de vida en el hábitat construido, para asegurar la existencia de una sociedad más equilibrada e incluyente (Bolivar Galvis (2012)).

- El estudio denominado El Uso Social del Espacio Público y su Vínculo con El Sistema Integrado de Transporte Masivo. Caso: Cartagena (Colombia), prioriza en la formulación de proyectos sobre la recuperación de los espacios públicos, porque contienen un alto impacto sobre el bienestar y la vida comunitaria de las poblaciones (Gonzales Saboya, Suarez, y Yori (2009)).

- En el estudio identificado como Proceso de Recuperación de Espacios Públicos de Municipio de Tuluá, Reubicación de Poblaciones de Vendedores Informales (Colombia), destacan la importancia al objetivo de la construcción de espacios como son plazas, zonas verdes, etc. Además, también enfatizan en la recuperación de los espacios abandonados, logrando que la gente se responsabilice de mantenerlos en buen estado y los use adecuadamente (Duarte, Aristizabal Valencia, y Carvajal Zapata (2008)).

- El estudio, Prevención Situacional del Delito en espacios Públicos Urbanos: Rol del Gobierno Local (Chile), plantea que para logra evitar los delitos en los espacios públicos urbanos, el gobierno local debe de tener un rol principal por ser una entidad política responsable de la toma de decisiones relacionadas al nivel territorial, pero esta función, el gobierno local, debe de realizarlo en conjunto con las comunidades que en él habitan (Salazar (s.f.)).

- El estudio, La Recuperación del Espacio Público para la Sociabilidad Ciudadana (Venezuela), considera que un espacio público recuperado tiene que cumplir con la condición de ser un espacio para la sociedad, favoreciendo un ambiente adecuado para qué las personas puedan realizar actividades que inciten a su evolución psicosocial, como ser. participar. recrearse, culturizarse y llegar a una plenitud espiritual(Rangel Mora (2002))

- El estudio, Espacios Públicos en la Frontera: Estudio Comparativo Ciudad Juárez Chihuahua - El Paso Texas (México - Estado Unidos), se realiza a partir de la perspectiva de una frontera como es el caso de Ciudad Juárez Chihuahua y El Paso Texas. Donde se busca identificar los factores que alteran la morfología urbana de las dos ciudades en relación con la configuración de sus espacios públicos analizando sus usos y características, pero siempre teniendo en cuenta el factor de ser ciudades fronterizas (Gutiérrez (2010))

De los estudios mencionados anteriormente, se destaca la postura de que al recuperar o construir espacios públicos, se puede generar un impacto de bienestar 
social, por ser un lugar de encuentro de diversos actores y cumplir la función de intercambio. También se resalta la importancia de los gobiernos locales en asegurar la existencia de estos espacios para la convivencia ciudadana.

En Honduras, En la Ley Marco del Desarrollo de la Juventud Decreto Número 260 - 2005, define sobre los derechos que tienen los jóvenes de tener un lugar donde puedan realizar actividades recreativas y de ocio. Estos artículos son: Artículo 6 y 13, promover una vida saludable e integral a través de la educación, la ciencia, la tecnología, el arte y la cultura general; el deporte, el ejercicio físico y otras actividades de sana recreación; los jóvenes tienen derecho a un nivel de vida digno y que la familia, la escuela, los órganos estatales y las organizaciones sociales promuevan la educación, la capacidad creativa, la cultura, el deporte y la recreación de los jóvenes (Congreso Nacional de Honduras. (2006)).

En el Reglamento General de la Ley de Municipalidades Acuerdo Número 018-93 en el Artículo 207 define: para la construcción, reconstrucción o modernización de plazas, parques jardines de recreo y canchas deportivas es de interés social decretar la expropiación de predios urbanos (Congreso Nacional de Honduras. (1993)).

\section{MEDOTOLOGÍA}

\section{III.1 El Alcance del Estudio}

El alcance del estudio es de tipo comparativo, analizando los patrones de distribución identificados en cada ciudad de estudio para identificar similitudes y diferencias.

\section{III.2 Profundidad del Estudio}

La investigación es cuantitativa; pues se procesan datos espaciales, clasificados y analizados cuantitativamente, se extraen patrones de distribución espacial, para ser comparados entre las ciudades de estudio.

\section{III.3 Temporalidad del Estudio}

El estudio es de corte transversal porque se realizó en un punto determinado en el tiempo para el año 2017.

\section{III.4 Unidad de Análisis y Población del Estudio}

La Población de análisis son las ciudades del Distrito Central, Comayagua y Siguatepeque y la unidad de análisis son los patrones de distribución espacial de las infraestructuras culturales, deportivas y recreativas de las ciudades.

\section{III.5 Clasificación de las Bases de Datos Existentes por Ciudad}

Se clasificaron las diferentes bases de datos de las infraestructuras de los espacios públicos en las categorías cultural, deportiva y recreativa para cada una de las ciudades. 


\section{III.6 Análisis de la Información}

Mediante el uso de herramientas SIG se definió el patrón para cada una de las ciudades y con análisis espacial se determinó la similitud y/o la diferencia de patrones entre las ciudades en estudio.

\section{Resultados}

\section{IV.1 Características de la Infraestructura de Espacios Públicos de la Ciudad del Distrito Central}

En la ciudad del Distrito Central existe un total de 374 infraestructuras para la oferta deportiva, cultural y recreativa de la ciudad, el $81 \%$ de la infraestructura es deportiva, el $10.7 \%$ es cultural y el $8.3 \%$ es recreativa (ver figura 1 ).

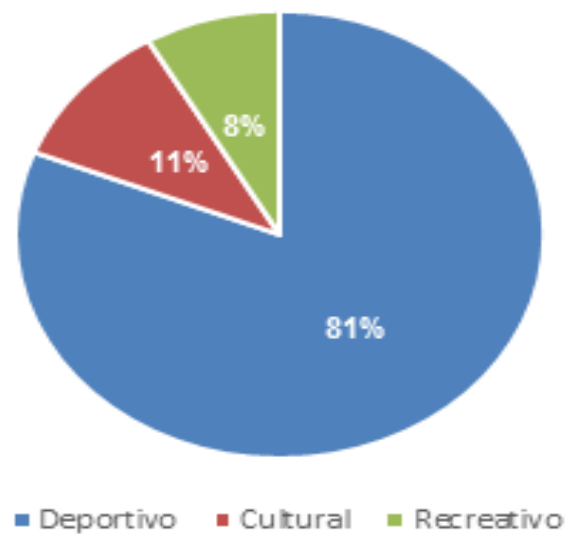

Figura 1: Distribución Porcentual de Espacios Públicos en la Ciudad del Distrito Central. Fuente: elaboración propia, (Sosa Caballero (2014)).

\section{IV.2 Características de la Infraestructura de Espacios Públicos de la Ciudad de Comayagua}

En la ciudad de Comayagua existe un total de 59 infraestructuras para la oferta deportiva, cultural y recreativa de la ciudad, el $64.4 \%$ de la infraestructura es deportiva, el $20.3 \%$ es cultural y el $15.3 \%$ es recreativo (ver figura 2 ). 


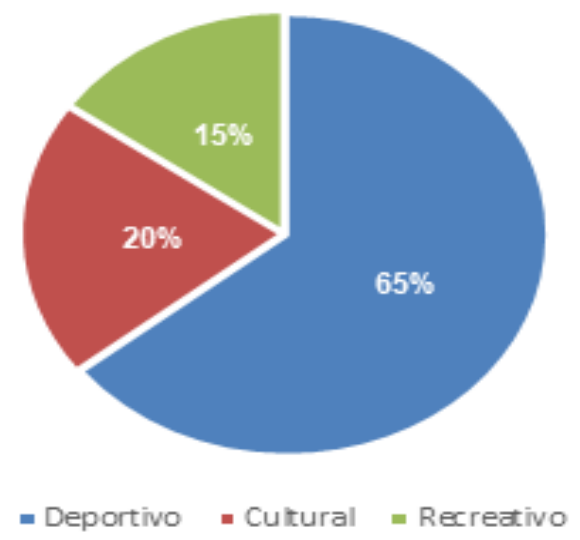

Figura 2: Distribución Porcentual de Espacios Públicos en la Ciudad de Coma-yagua. Fuente: elaboración propia, (Sosa Caballero (2016)).

\section{IV.3 Características de la Infraestructura de Espacios Públicos de la Ciudad de Siguatepeque}

En la ciudad de Siguatepeque existe un total de 58 infraestructuras para la oferta deportiva, cultural y recreativa de la ciudad, el $75.9 \%$ de la infraestructura es deportiva, el $8.6 \%$ es cultural y el $15.5 \%$ es recreativo (ver figura 3 ).

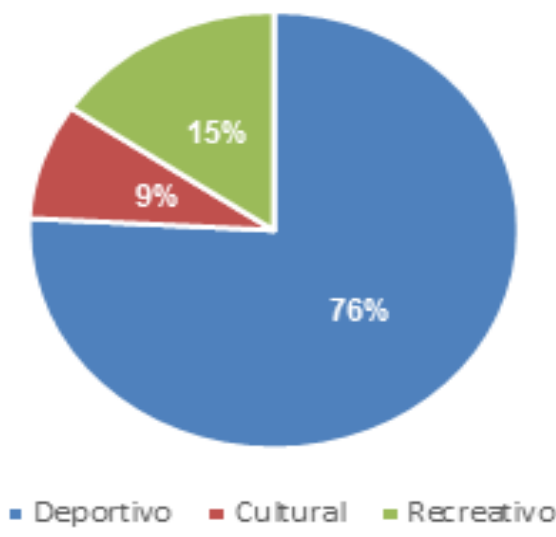

Figura 3: Distribución Porcentual de Espacios Públicos en la de Siguatepeque. Fuente: elaboración propia, (Sosa Caballero (2016)).

\section{IV.4 Patrón de Distribución Espacial de Espacios Públicos en las Tres Ciudades}

Las ciudades en estudio con relación a la infraestructura deportiva poseen más instalaciones para las categorías de canchas de fútbol de colonia o barrio, canchas de futbolito privado y canchas de baloncesto (ver figura 4). 


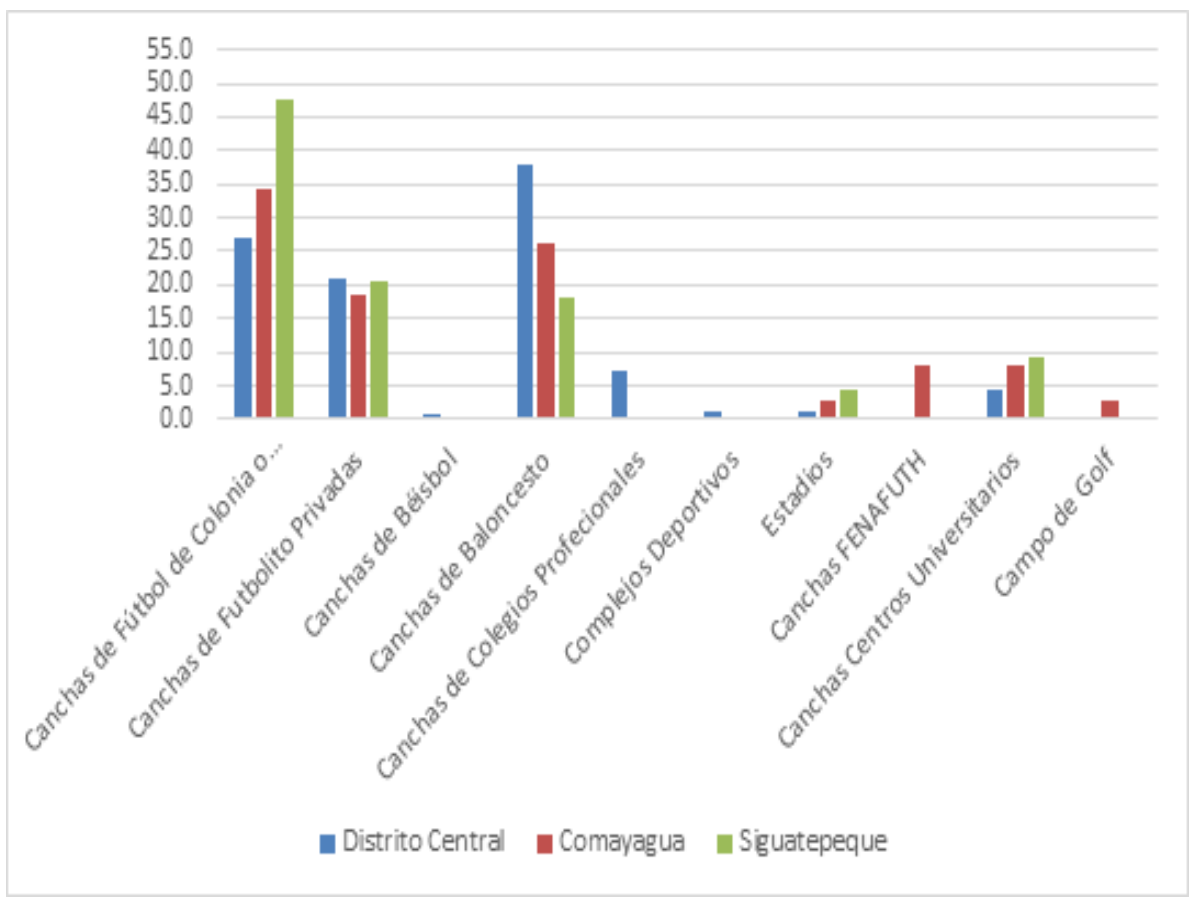

Figura 4: Comparación de la Infraestructura Deportiva en las Tres Ciudades. Fuente: elaboración propia.

La distribución espacial de las infraestructuras deportivas, las ciudades poseen un patrón similar de distribución de las instalaciones por toda la ciudad (ver figuras 5,6 y 7 ). 
Análisis de la distribución espacial de los espacios públicos en las ciudades del Distrito Central, Comayagua y

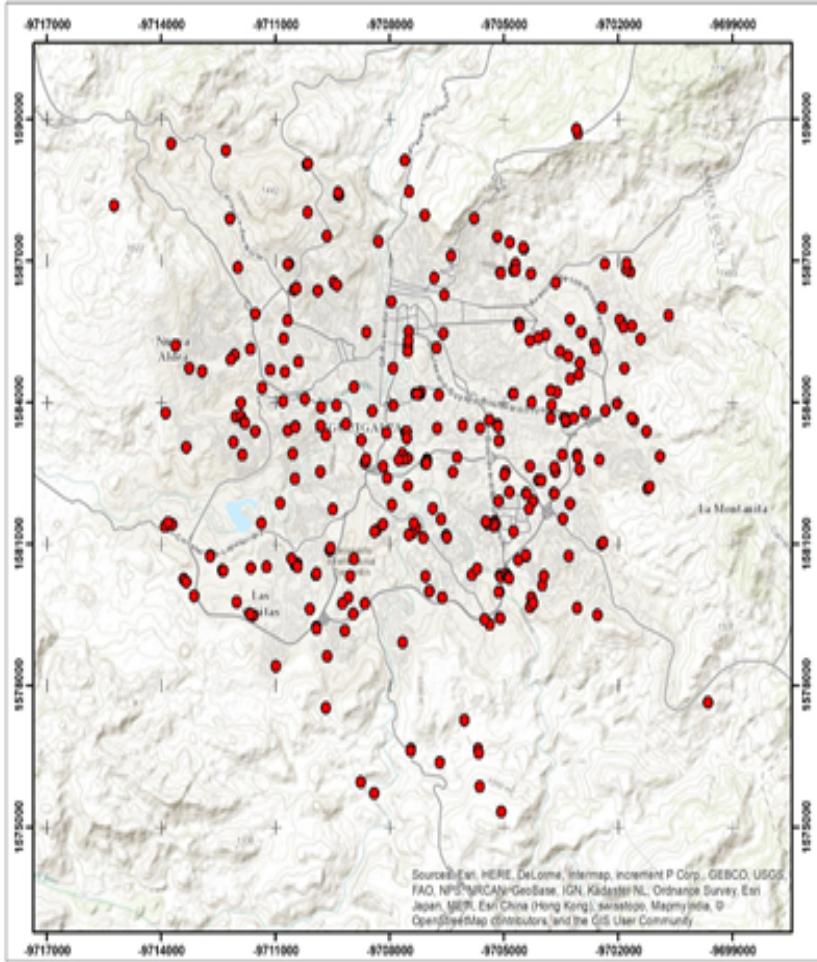

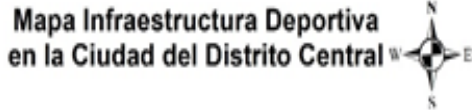

Fescala 1:100,000

5.5 2.75

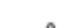$$
\text { (n) }
$$

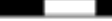

Proyección UTM Zona 16 Norte Elipsoide WGSBA

\section{Simbologia}

- Infraestructura Deportiva

Fuente: Elaboración Propia, Levartamiento de cras para el Proyecto Distribución de los Espacios Públicos en el Distrito Central de Honduras, para el Anslisis del Equiltorio Territorial de la Oterta Cultural y Recreativa de la Cludad y utizando imagen de World Topographic Map de Esni, HERE, Delome, Intermap. increment P Corp, GEBCO, USGS, FAO, NPS, NRCAN

GecBase, ICN Kadaster NL. Ordnance Survey,

Essi Japan, METI, Esri China (Hong Kong), swisstopo Mapmylnda, OoenStreetMab Contributors and the GIS User Community

tuborado Por: ing. Celina Michelie Scas Caballero Junip del 2017.

Figura 5: Mapa de Ubicación Infraestructura Deportiva en la Ciudad del Distrito Central. 


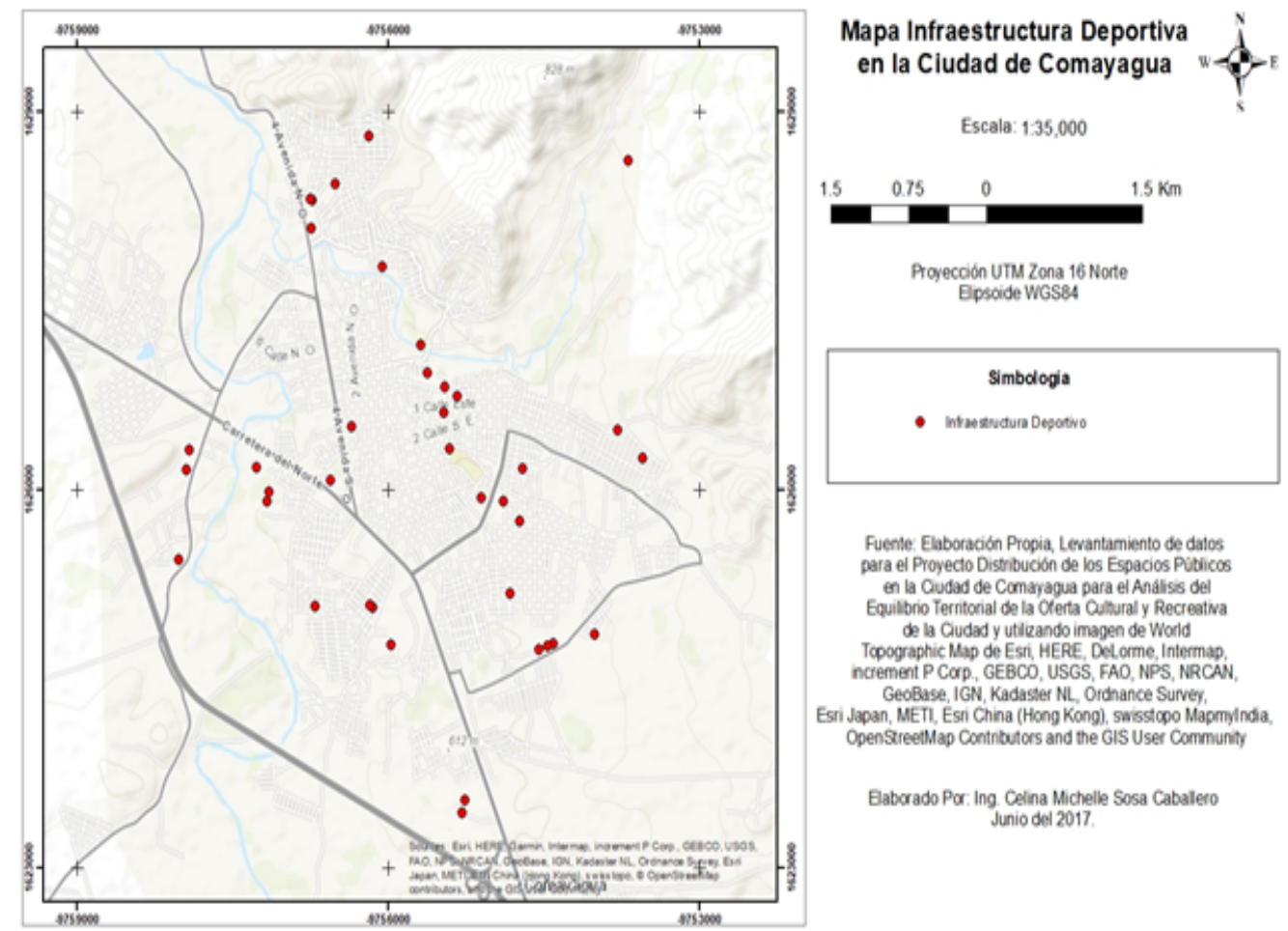

Figura 6: Mapa de Ubicación Infraestructura Deportiva en la Ciudad de Comayagua. 
Análisis de la distribución espacial de los espacios públicos en las ciudades del Distrito Central, Comayagua y

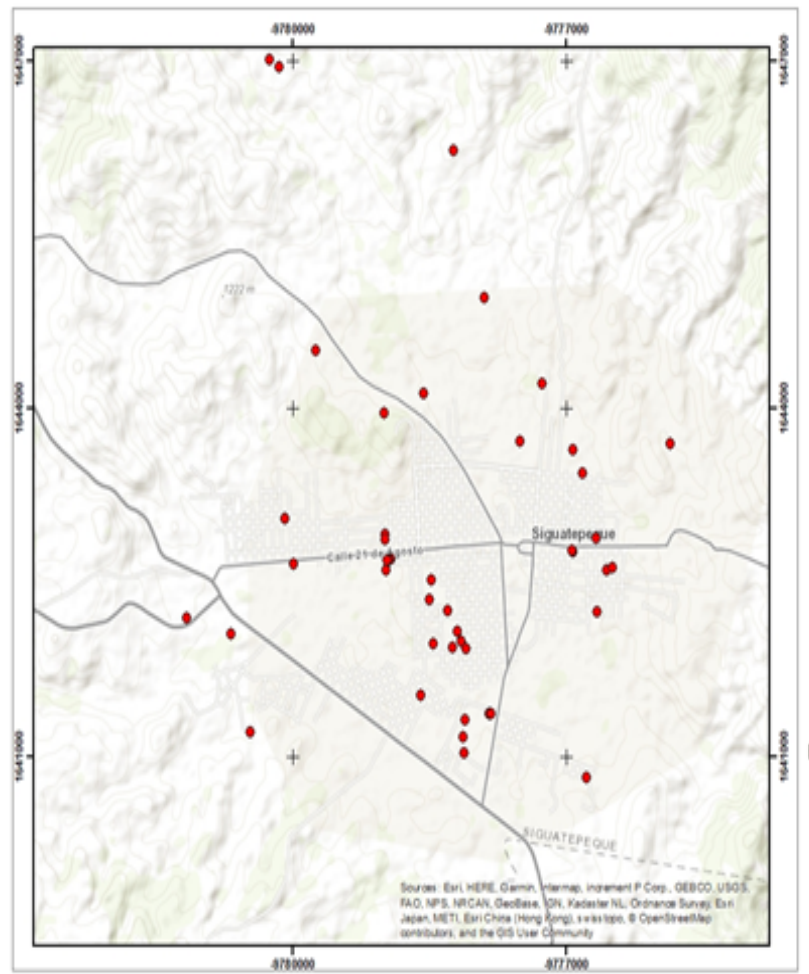

Mapa Infraestructura Deportiva en la Ciudad de Siguatepeque

Escala: $1: 40,000$

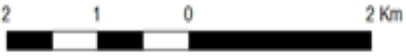

Proyección UTM Zona 16 Norte Elpsoide WGS84

Simbologia

- intas atrudura Degotiva

para el proyecto Distrbución de los Espacios Publicos on la Cudad de Siguatepeque para el Analisis del Equilbeno Ternitorial de la Ofenta Cultural y Recreativa

de la Cudad y ublizando imagen de Wond

Topographic Map de Esri. HERE Delorme Intermap

increment P Cap, GEBCO, USGS, FAO, NPS, NRCAN

Geobase, IGN, Kadaster NL, Ordnance Survey.

Esti Japan, METI, Esti China (Hong Kong) swisstcoo Mapmylndia,

OpenStreesMap Contributors and the GIS User Communty

Baborado Por. Ing Celina Michelle Sosa Cabalero

Junio del 2017

Figura 7: Mapa de Ubicación Infraestructura Deportiva en la Ciudad de Siguatepeque.

En la infraestructura cultural representada en la figura 8 se puede observar que mayor número de instalaciones corresponde a bibliotecas y cines; además las instalaciones de museos que se observan se encuentran en las ciudades del Distrito Central y Comayagua. 


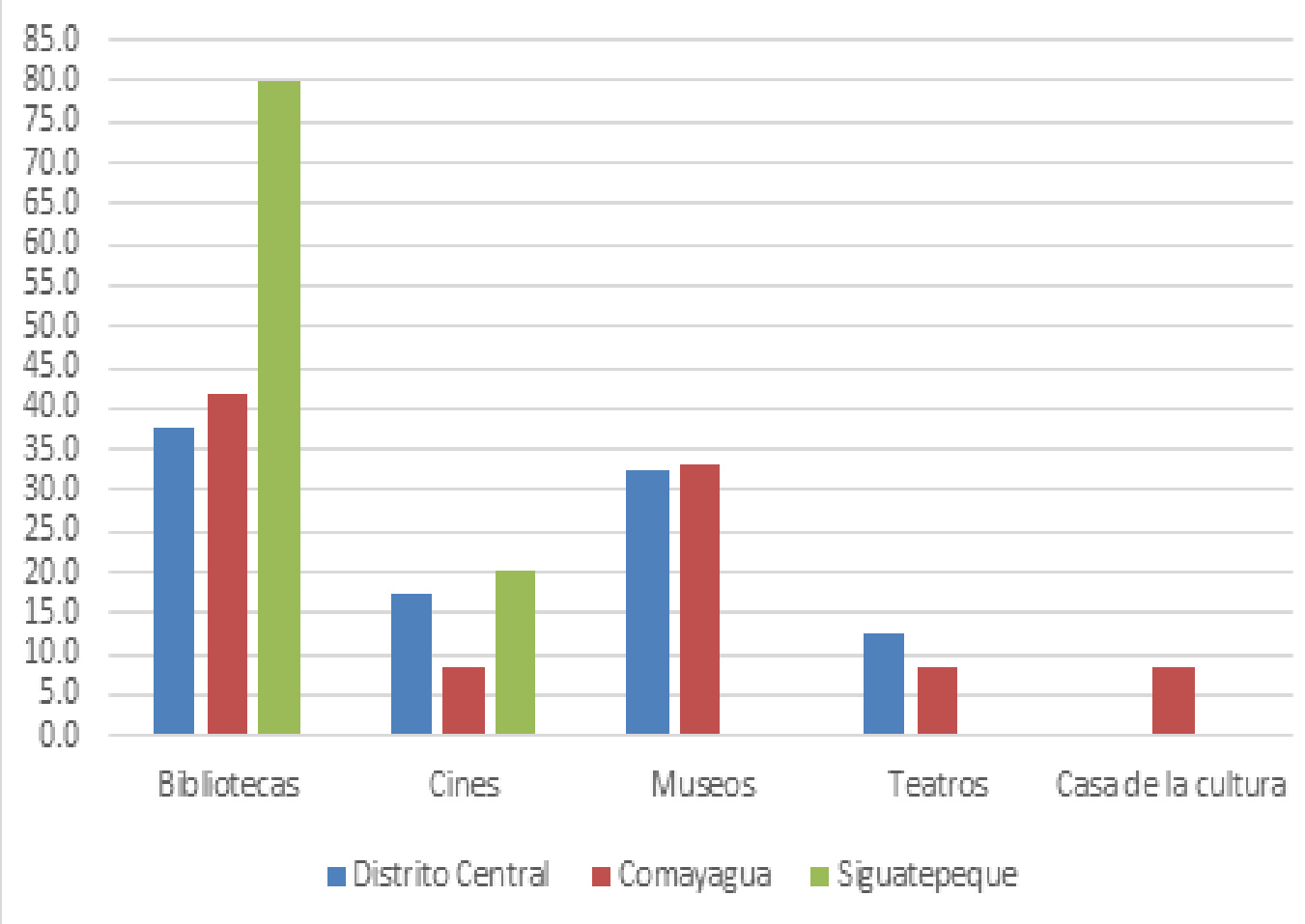

Figura 8: Comparación de la Infraestructura Cultural en las Tres Ciudades. Fuente: elaboración propia.

La distribución espacial de la infraestructura cultural, en las ciudades del Distrito Central y Comayagua tiene un patrón similar en la concentración de la infraestructura cultural en el centro de cada una de las ciudades (ver figuras 9, 10 y 11). Mientras que en la ciudad de Siguatepeque se observa solamente cinco instalaciones para infraestructura cultural frente a las 40 y 12 instalaciones de este tipo de infraestructura observada para la ciudad del Distrito Central y la de Comayagua respectivamente. 
Análisis de la distribución espacial de los espacios públicos en las ciudades del Distrito Central, Comayagua y Siguatepeque, Honduras, 2017

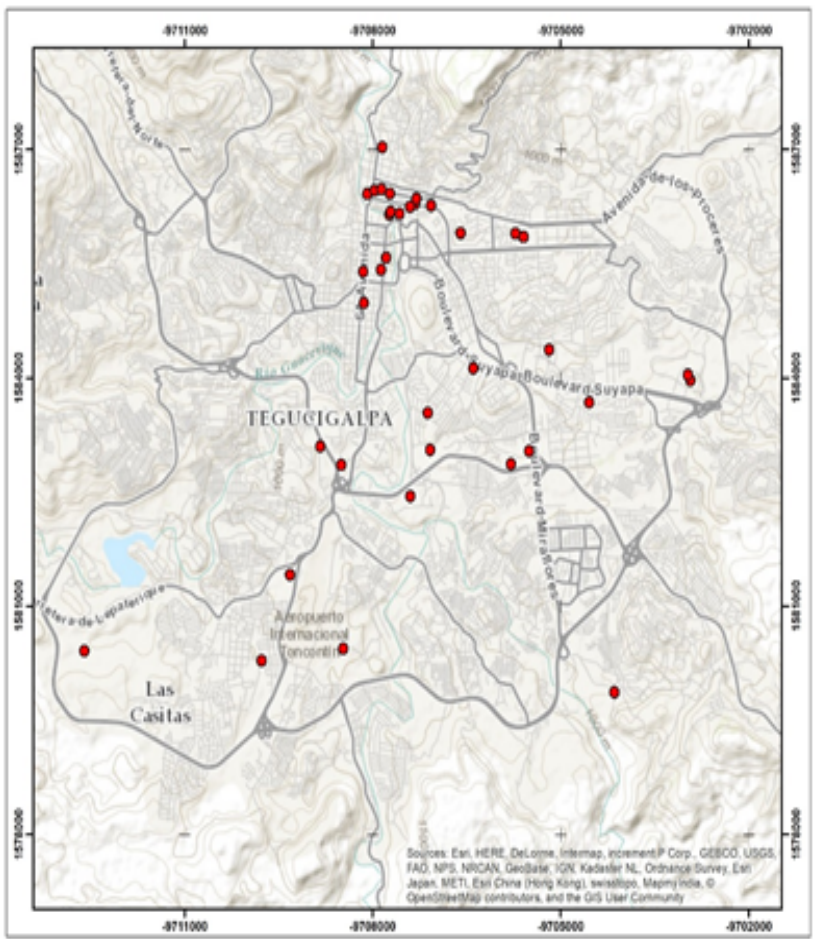

Mapa Infraestructura Cultural en la Ciudad del Distrito Central "

Escala: $1 ; 60,000$

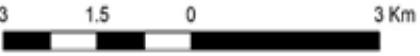

Proyección UTM Zona 16 Norte Elipsoide WGS84

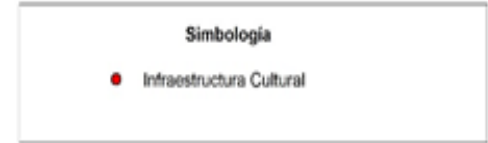

Fuente. Elaboración Propia, Levantamiento de dalos para et Proyecto Destribuoión de los Espacios Publicos en el Distrto Central de Honduras, para el Análsis del Equibrio Teritorial de la Oterta Qumaral y Recres:iva de la Cudad y uticando imagen de World

Topogaphic Map do Esri HERE, Delorme. Intermap. nocement P COOP GEBCO USGS. FAO NPS, NRCAN, Geobase ICN Kadaster NL Ondnance Suney.

Esri Japan, METI, Esti China (Hong Kongl. swisstopo Mapmyloda CpensieetMap Controutors and the GiS User Communty

Eaborado Por: Ing Colina Michelle Sosa Cabalero Iinio del 7017

Figura 9: Mapa de Ubicación Infraestructura Cultural en la Ciudad del Distrito Central. 


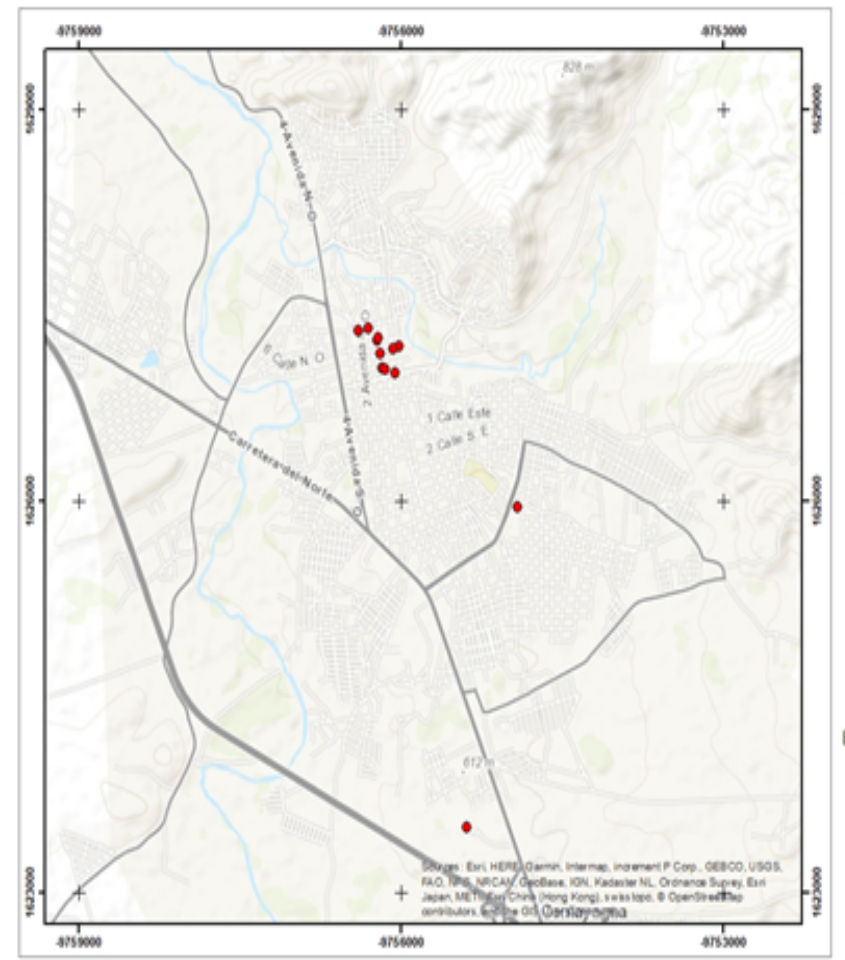

Mapa Infraestructura Cultural

en la Ciudad de Comayagua

Escala: $1: 35,000$

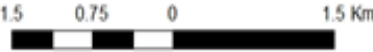

Proyección UTM Zona 16 Norte Elpsoide WGS84

Simbologia

- Infraestructura Cutural

Fuente Elaboracón Propia Levantamiento de datos para el Proyecto Distribución de los Espacios Púbicos en la Cudad de Comayagua para el Analisis del Equilbrio Territorial de la Clerta Qultural y Recreativa de la Qudad y utizando imagen de World

Topographic Map de Esn, HERE, DeLorme, Intermap. increment P COrP, GEBCO, USGS, FAO, NPS, NRCAN

GeoBase, IGN Kadaster NL, Ordnance Surver. Esri Japan METI, Esn China (Hong Kong). swisstopo Mapmyinda OpenstreetMap Contributors and the GIS User Community

Baborado Por: Ing Celina Michelle Sosa Cabalero Junio del 2017

Figura 10: Mapa de Ubicación Infraestructura Cultural en la Ciudad de Comayagua. 
Análisis de la distribución espacial de los espacios públicos en las ciudades del Distrito Central, Comayagua y Siguatepeque, Honduras, 2017

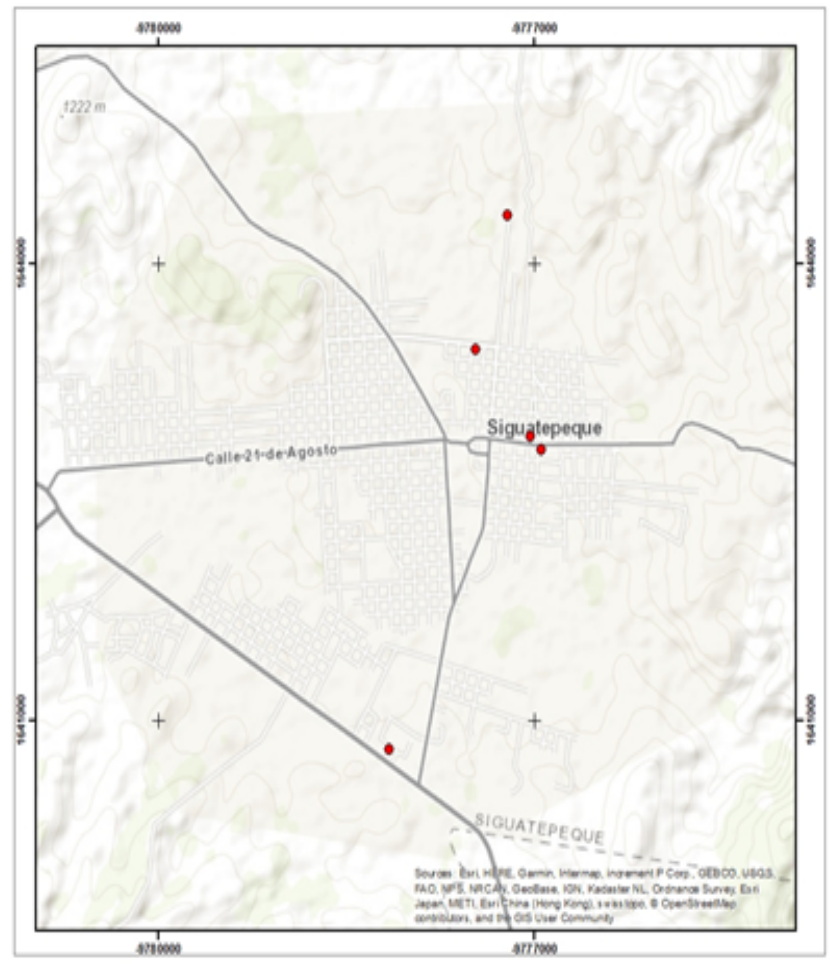

\section{Mapa Infraestructura Cultural \\ en la Ciudad de Siguatepeque}

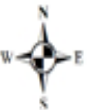

Escala: 130,000

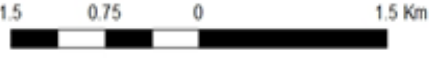

Proyección UTM Zona 16 Norte Epsoide WGS8

Simbologia

- Intas trvdura Culura

Fuente: Levantamiento de datos para el proyecto Distrbución de los Espacios Publicos en la Cudad de Siguatepeque para el Análisis del Equilberio Termonial de la Olenta Cultural y Recreativa de la Cudad y uthizando imagen de World Topographic Map de Esri, HERE, Delorme, Intermap, nctement P Cap, GeoBase, IGN Kadister Esti Japan, METI, Esri China (Hong Kongl, swssicopo Mapmylndia, OpenStreedMap Contributors and the GIS User Communty

Baborado Por. Ing Celina Michelle Sosa Caballero Junio del 2017

Figura 11: Mapa de Ubicación Infraestructura Cultural en la Ciudad de Siguatepeque.

Respecto a la infraestructura recreativa en las ciudades objeto estudio se observa que sobresalen los parques y plazas (ver figura 12). 


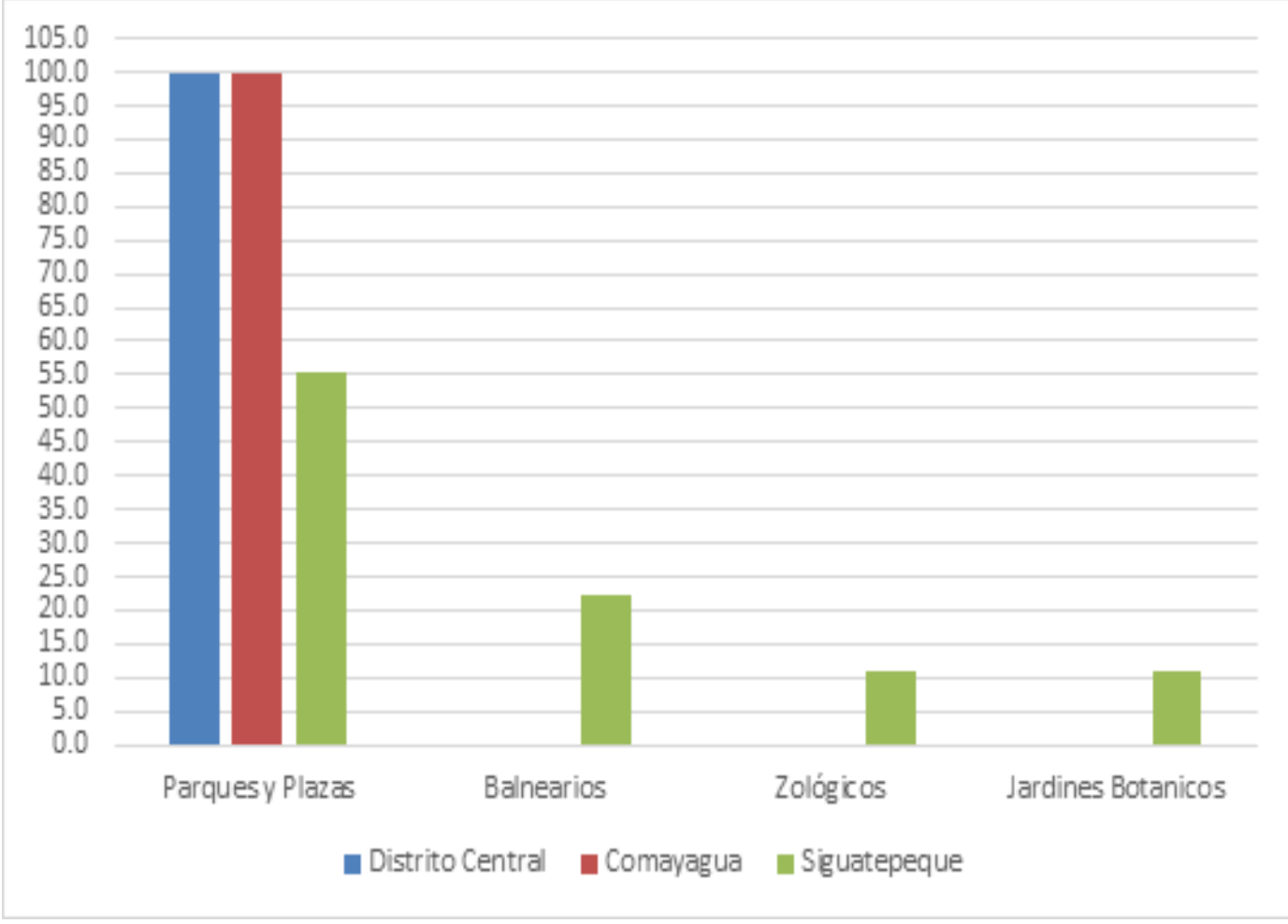

Figura 12: Comparación de la Infraestructura Recreativa en las Tres Ciudades. Fuente: elaboración propia.

En relación con la distribución espacial de la infraestructura recreativa, las tres ciudades tienen un patrón similar caracterizado por la concentración de instalaciones en el centro de cada ciudad y unas pocas instalaciones distribuidas en el resto de las mismas (ver figuras 13,14 y 15 ). 
Análisis de la distribución espacial de los espacios públicos en las ciudades del Distrito Central, Comayagua y Siguatepeque, Honduras, 2017

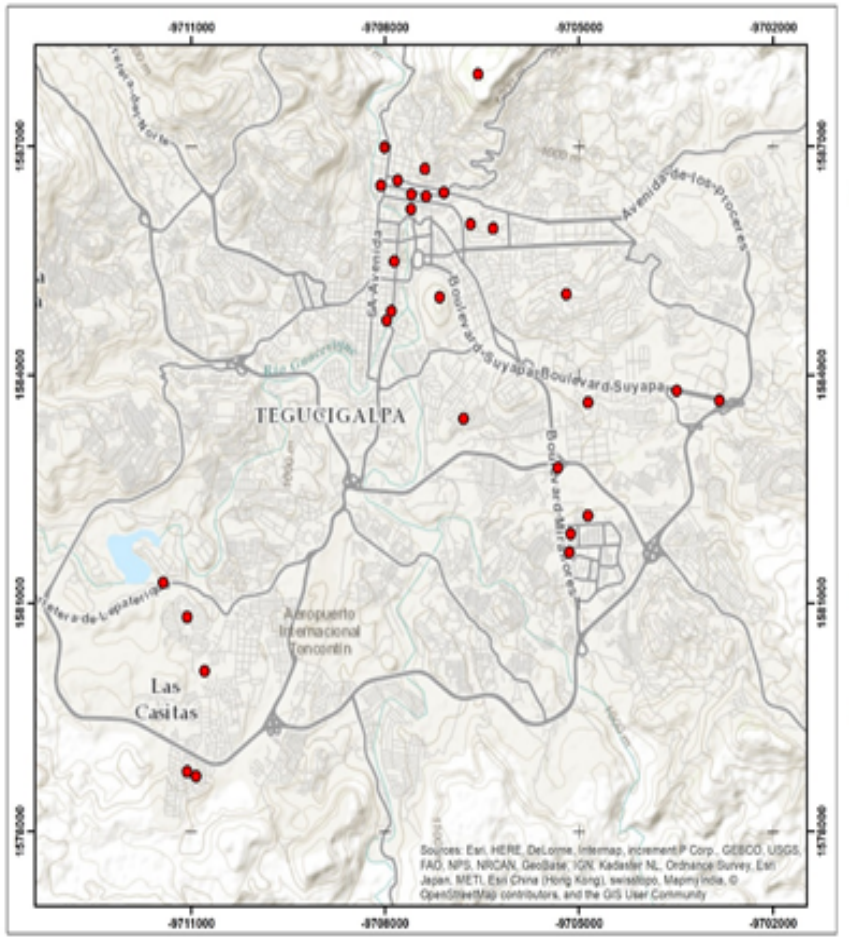

Mapa Infraestructura Recreativa en la Ciudad del Distrito Central

Escala: $1: 60,000$

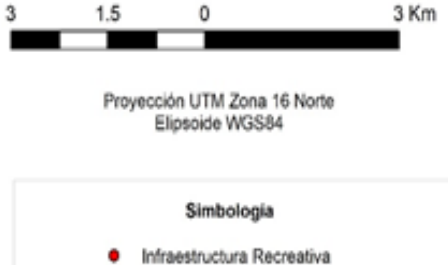

Fuente. Elaboracón Propia Levantamiento de datos para ef Proyecto Distrbución de los Espacios Publicos en el Distrto Central de Honduras, para el Análisis del Equilbrio Terntorial de la Oferta Cullural y Recrea'iva de la Cudad y utizando imagen de World Topographic Uap do Esri. HERE, Delorme, Intermap. ncrement P Corp. GEBCO, USGS, FAO, NPS, NRCA GeoBase, IGN Kadaster NL, Orchance Survey. Esri Japan, METI, Esri China (Hong Kong). swisstopo Mapmylnda, OpenStreedMap Contributors and the GiS User Communty

Eaborado Por: Ing Celina Michelle Sosa Cabalevo Junio del 2017.

Figura 13: Mapa de Ubicación Infraestructura Recreativa en la Ciudad del Distrito Central. 


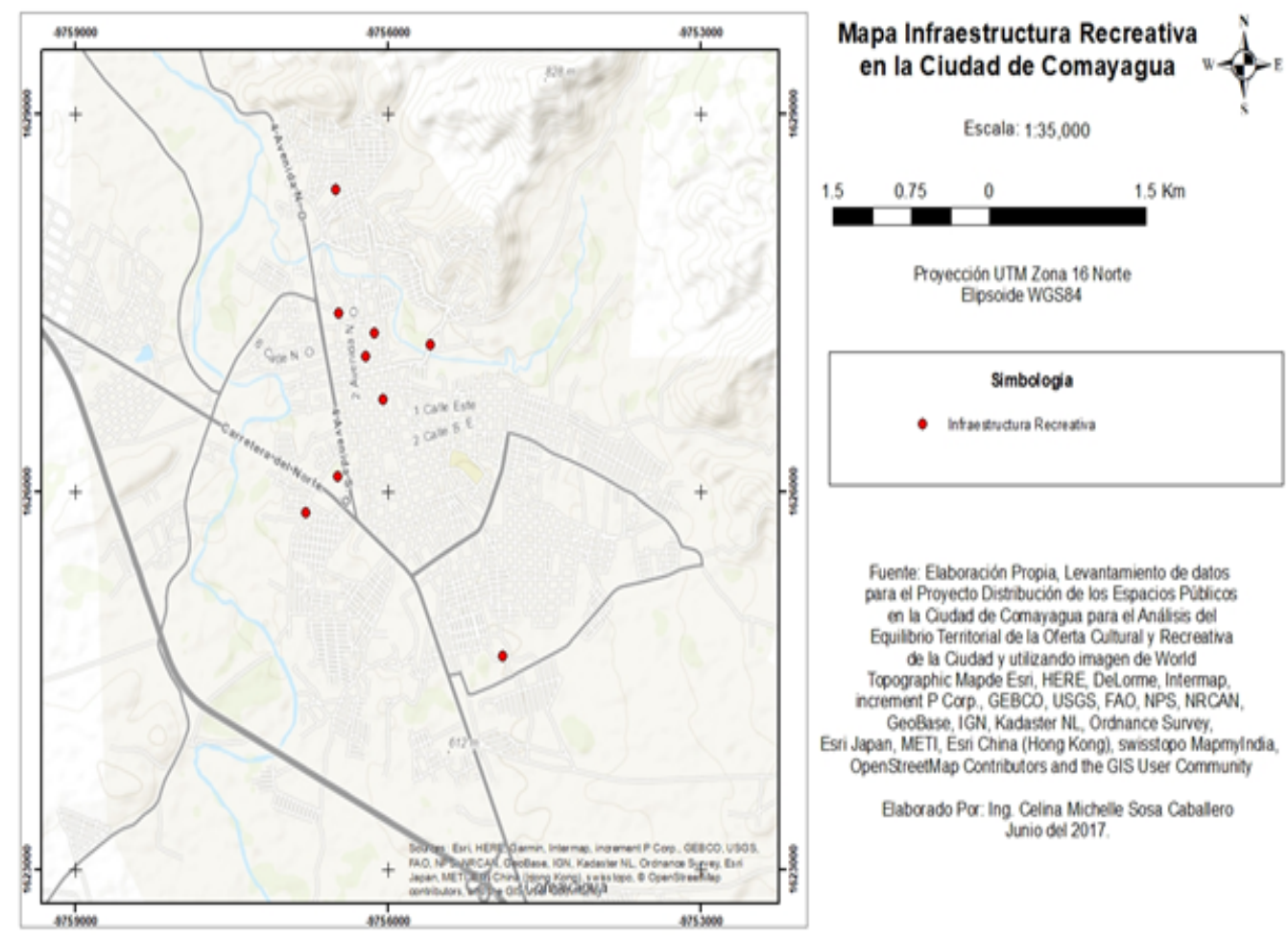

Figura 14: Mapa de Ubicación Infraestructura Recreativa en la Ciudad de Comayagua. 
Análisis de la distribución espacial de los espacios públicos en las ciudades del Distrito Central, Comayagua y Siguatepeque, Honduras, 2017

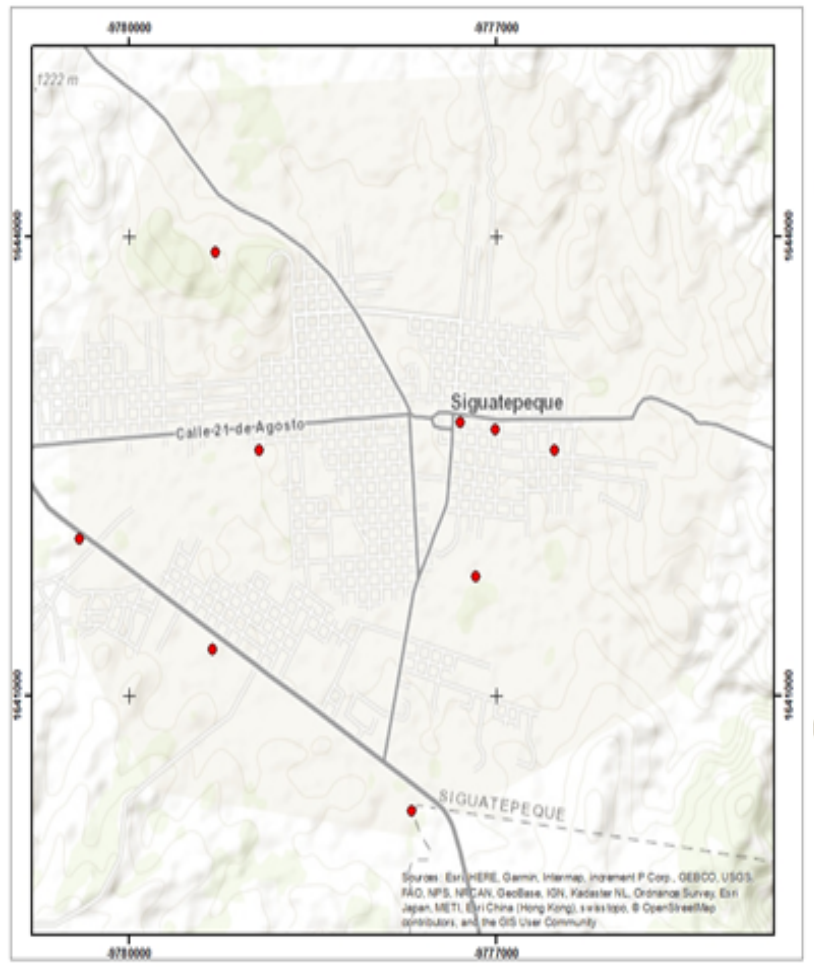

Mapa Infraestructura Recreativa en la Ciudad de Siguatepeque

Escala: $1: 30,000$

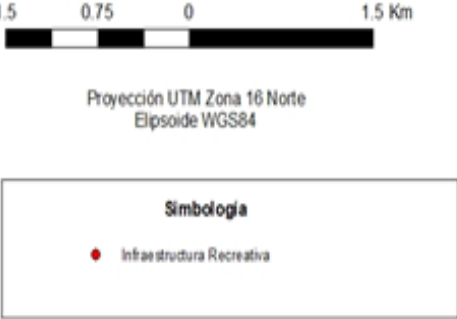

Fuente: Levantamiento de datos para el proyecto Distribución de los Espacios Púbicos en la Cudad de Siguatepeque para el Análisis del Equilibrio Territorial de la Oterta Cultural y Recreativa de la Cudad y utilizando magen de Work Topographic Map de Esri. HERE, DeLome, Intermap. increment P COrP, GEBCO, USGS, FAO NPS, NRCAN, GeoBase, IGN, Kadaster NL, Ordnance Survey. Esri Jipan, METI, Essi China (Hong Kong), swisstcoo Mapmyinda OpenStreetMap Contributors and the GIS User Community

Baborado Por: Ing Celina Michele Sosa Cabalero Junio del 2017

Figura 15: Mapa de Ubicación Infraestructura Recreativa en la Ciudad de Siguatepeque. 


\section{IV.5 Relación de Área de Espacios Públicos de Ciudades en Estudio frente al Parámetro de la Organización Mundial de la Salud}

Al hacer la relación de las ciudades en estudio con el parámetro recomendado por la Organización Mundial de la Salud (OMS) de un mínimo de $10^{2}$ de área verde por habitante dispersado equitativamente en relación a la población (CATMED. (2018)), la ciudad del Distrito Central representa el $47 \%$, la ciudad de Comayagua el $20 \%$ y la ciudad de Siguatepeque representa el $151 \%$ (ver figura 16).

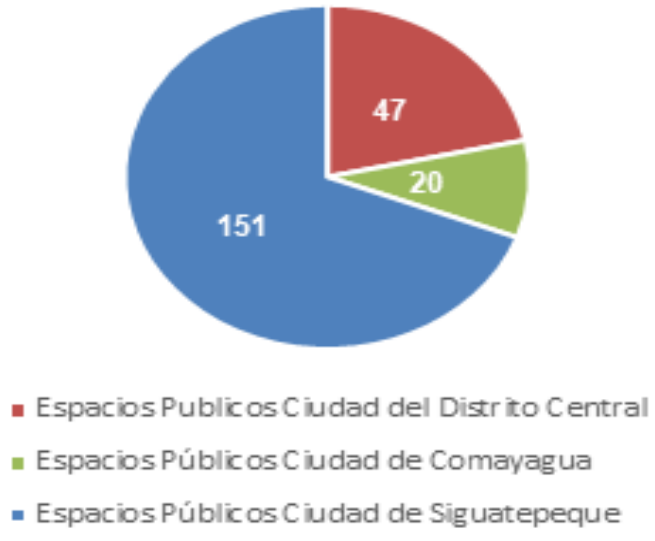

Figura 16: Distribución en Porcentajes en Relación de las Área de Espacios Públicos de Ciudades en Estudio frente al Parámetro de la OMS. Fuente: elaboración propia.

\section{CONCLUSIONES}

Una primera tendencia es el patrón de distribución espacial de la infraestructura deportiva; una segunda tendencia es el patrón de concentración espacial de la infraestructura cultural y recreativa.

La distribución espacial de los espacios públicos en las tres ciudades presenta similitud en la categoría de infraestructura deportiva y recreativa, y diferencia en la categoría cultural.

Con el estudio "Análisis de la Distribución Espacial de los Espacios Públicos en las Ciudades del Distrito Central, Comayagua y Siguatepeque, Honduras" se espera sea una contribución para investigadores interesados en el tema y sirva de referencia para los tomadores de decisiones de las ciudades estudiadas.

\section{Referencias}

Bolivar Galvis, A. M. (2012). Espacio público y calidad urbana en pereira visión de una nueva ciudad. Pereira, Colombia: Universidad Católica de Pereira, Facultad de Arquitectura y Diseño Industrial . 
CAT-MED. (2018). Zonas verdes y Áreas de esparcimiento. Plataforma para modelos urbanos sostenihles nescargado de http://www.catmed.eu/dic/es/50/zonas-verdes-y-areas -de-esparcimiento

CCVAH. (2018). Agenda de ordenamiento territorial del concejo centroaméricano de vivivenda y asentamientos humanos. El Salvador.

Ciudades y Gobiernos Locales Unidos, c. (2004). Comisión de cultura, agenda 21 de la cultura. Barcelona, España..

Congreso Nacional de Honduras., c. (1993). Reglamento general de la ley de municipalidades. Tegucigalpa, Honduras..

Congreso Nacional de Honduras., c. (2006). Ley marco para el desarrollo integral de la juventud. Tegucigalpa, Honduras..

Duarte, T., Aristizabal Valencia, M. A., y Carvajal Zapata, R. (2008). Proceso de recuperación de espacios públicos del Municipio de Tula - reubicación de población de vendedores informales. Pereira, Colombia: Universidad Tecnólogica de Pereira, Facultad de Ingeniería Industrial.

Gonzales Saboya, S. C., Suarez, G., y Yori, C. M. (2009). El uso social del espacio público y sus vinculos con el sistema integrado de transporte masivo. Cartagena . Bogotá. Colombia: Pontificia Universidad Javeriana Facultad de Arquitectura y Diseño Maestría en Planeación Urbana y Regional.

Gutiérrez, L. (2010). Espacios públicos en la frontera: Estudio comparativo Ciudad Juárez Chihuahua - El Paso Texas. Guadalajara Jalisco, México.

Madanipour, S., A.and Knierbein, y Degros, A. (2014). Políticas para el espacio público en las ciudades europeas.

Rangel Mora, M. (2002). La recuperación del espacio público para la sociabilidad ciudadana. Venezuela: Universidad de Los Andes, Facultad de Arquitectura y Arte, Programa del Posgrado Desarrollo Urbano Local.

Salazar, F. (s.f.) La prevención situacional del delito en espacios públicos urbanos: Rol del gobierno local. seguridad y violencia. Desafios para la Ciudadanía,.

Sosa Caballero, C. M. (2014). Distribución geoespacial de los espacios públicos en el Distrito Central de Honduras

Sosa Caballero, C. M. (2016a). Distribución de los espacios públicos en la ciudad de Siguatepeque para el análisis del equilibrio territoial de la oferta cultural y recreativa de la ciudad. Tegucigalpa, Honduras: sin publicar.

Sosa Caballero, C. M. (2016b). Distribución geoespacial de los espacios públicos en la ciudad de Comayagua, Honduras. 